\title{
Infracomunidades de parásitos eumetazoos del bagre de mar Aphos porosus (Valenciennes, 1837) (Actinopterygii: Batrachoidiformes) en Chile central
}

\author{
Eumetazoan parasite infracommunities of the toadfish Aphos porosus (Valenciennes, 1837) \\ (Actinopterygii: Batrachoidiformes) from central Chile \\ Yurima Cortés ${ }^{1}$ y Gabriela Muñoz ${ }^{1}$ \\ ${ }^{1}$ Facultad de Ciencias del Mar y de Recursos Naturales, Universidad de Valparaíso, Valparaíso, \\ Casilla 5080, Reñaca, Viña del Mar, Chile \\ y90210ch@yahoo.com
}

\begin{abstract}
This study describes and quantifies the eumetazoan parasite community of the toadfish Aphos porosus (Valenciennes, 1837). To accomplish this purpose, 30 toadfish individuals were collected from intertidal rocky pools at El Tabo, Central Chile between October 2006 and January 2007. The descriptors of the parasite infracommunity (abundance, richness, diversity and eveness) were calculated and correlated with the host body weight to determine if these variables have any relationship. The trophic level in which the toadfish is positioned in its community is also suggested, according to the life cycles of the parasites found in the fish. From the total sample, 93\% fish were parasitized and 2,104 parasite individuals, belonging to nine taxa, were collected. The cestodes of Tetraphyllidea and the nematode Pseudoterranova sp. were the most prevalent and abundant parasites. At the infracommunity level, only the abundances (numeric and volumetric) of parasites increased significantly with host body weight which may be a consequence of a major consumption of infected prey and accumulation of parasites because several parasite species were larvae and encysted in the celomatic cavity and muscles of the fish. At the infrapopulation level only Tetraphyllidea showed a significant correlation between abundances and host body size. The toadfish would be in an intermediate trophic level; it preys over crustaceans which are intermediate hosts for several parasite species found in this study. At the same time, toadfish can be preyed by elasmobranchs and marine mammals which usually are definitive hosts of most endoparasite species found.
\end{abstract}

Key words: Life cycle, parasite communities, intertidal zone, parasite transmission
Resumen.- En este estudio se describe y cuantifica la comunidad de parásitos eumetazoos del bagre de mar Aphos porosus (Valenciennes, 1837). Con este objeto se recolectaron 30 ejemplares desde las pozas intermareales de El Tabo, Chile central, entre octubre 2006 y enero 2007. Se cuantificaron los descriptores infracomunitarios de parásitos (riqueza, abundancia numérica, abundancia volumétrica, dominancia, diversidad y uniformidad) y se correlacionaron con el peso corporal del hospedador para determinar si había relación entre estas variables. Se indagó sobre el nivel trófico que ocupa el bagre de mar en su comunidad de acuerdo a los ciclos de vida de los parásitos encontrados en este hospedador. El 93\% de los bagres de mar estaba parasitado. Se recolectaron 2.104 individuos parásitos, pertenecientes a nueve taxones. Los cestodos de Tetraphyllidea y el nemátodo Pseudoterranova sp. fueron los parásitos más prevalentes y abundantes. A nivel infracomunitario sólo las abundancias (numérica y volumétrica) de parásitos aumentaron significativamente con el tamaño corporal del bagre de mar, lo que podría ser causa de una mayor ingesta de presas infectadas y a una acumulación de parásitos a medida que el hospedador crece, dado que varias especies parásitas se encontraron en estados larvales enquistados en la cavidad celomática y musculatura del pez, mientras que a nivel poblacional, sólo Tetraphyllidea aumentó en abundancia con el tamaño corporal del hospedador. El bagre de mar se ubicaría en un nivel trófico intermedio; depredaría sobre crustáceos, los cuales son hospedadores intermediarios de varias de sus especies parásitas, y a su vez, sería consumido por elasmobranquios y mamíferos marinos, principales hospedadores definitivos de la mayoría de los endoparásitos registrados.

Palabras clave: Ciclos de vida, comunidades de parásitos, zona intermareal, transmisión de parásitos

\section{Introducción}

En Chile, los estudios parasitológicos han ido en aumento en las últimas tres décadas, en especial aquellos referentes a peces como hospedadores (Muñoz \& Olmos 2005¹). Esto se debe a que los parásitos no sólo son unidades de estudio interesantes sino que además constituyen herramientas útiles para develar problemas biológicos. Por ejemplo, los estudios parasitológicos ayudan en la investigación pesquera a la identificación de unidades de stock (George-Nascimento \& Arancibia 1994, Oliva \& 
Ballón 2002), patrones de migración y de reclutamiento de juveniles (Oliva et al. 2004) y patrones zoogeográficos de determinados recursos (González \& Moreno 2005, Oliva \& González 2005, González et al. 2006). Asimismo, mediante los análisis parasitológicos, es posible determinar los eslabones tróficos entre los hospedadores y otros organismos, el nivel trófico del hospedador y los cambios ontogenéticos de la dieta (Marcogliese 2003), aunque este último tema ha sido escasamente tratado en Chile (George-Nascimento 1987).

En vertebrados se han realizado muchas investigaciones sobre parásitos a nivel de infracomunidad, que corresponde a todas las poblaciones de las distintas especies de parásitos dentro de un individuo hospedador (Bush et al. 1997). Esto se debe a que los estudios infracomunitarios constituyen la base de cualquier trabajo parasitológico y son útiles para hacer comparaciones con otras poblaciones de un hospedador particular o entre especies de hospedadores por medio de sus descriptores parasitarios. De hecho, en Chile, los peces cuentan con numerosos estudios parasitológicos, aunque gran parte de ellos han sido sobre registros y descripciones taxonómicas de especies parásitas (Muñoz \& Olmos 2005 ${ }^{1}$ ). Sin embargo, aún quedan muchas especies de este grupo en las que no se ha analizado la composición comunitaria de parásitos ni se han descrito cuantitativamente sus comunidades, como es el caso de varias especies de peces intermareales. Dentro de este grupo, el 'bagre de mar', Aphos porosus (Valenciennes, 1837), es un hospedador interesante desde el punto de vista ecológico, ya que tiene la capacidad de desplazarse desde el ecosistema demersal somero sobre fondos blandos de Chile (Sielfeld \& Vargas 1996) a zonas intermareales y estuarinas (Moreno \& Castilla 1975) en primavera durante su época reproductiva (Varas \& Ojeda 1990). Esta característica de pez transitorio del intermareal (Varas \& Ojeda 1990) lo hace un transmisor potencial de parásitos desde el ambiente demersal al intermareal y viceversa, en cuyo trayecto puede incorporar o perder especies de parásitos.

La especie $A$. porosus es la única de la familia Batrachoididae que la literatura registra en Chile (Pequeño 1989). Su distribución se extiende en el Océano Pacífico sudamericano desde Puerto Pizarro en Perú hasta Magallanes en Chile (Chirichigno 1974), donde usualmente cohabita con Sebastes capensis (Gmelin, 1789), Prolatilus jugularis (Valenciennes, 1833) y especies del género Merluccius (Moreno \& Castilla 1975, Angel \&

\footnotetext{
${ }^{1}$ Muñoz G \& V Olmos. Parasitología en sistemas acuáticos de Chile: una revisión de lo publicado. FLAP XVII, 2005. Mar del Plata. Argentina.
}

Ojeda 2001). El bagre de mar alcanza aproximadamente 28 cm de longitud total (Medina et al. 2004) y se ha determinado como una especie carnívora estricta que se alimenta de gasterópodos, bivalvos, peces óseos y crustáceos decápodos (Varas \& Ojeda 1990). Sólo se ha realizado un estudio de parásitos en esta especie, el cual registró cinco taxones de helmintos parásitos: el cestodo Scolex pleuronectis Müller, 1788; los nemátodos Anisakis simplex (Rudolphi, 1809), Pseudoterranova decipiens (Krabbe, 1878) y Capillaria sp. y el acantocéfalo Corynosoma sp. (Torres et al. 1993).

Este estudio tuvo como principal objetivo, describir y cuantificar las infracomunidades de parásitos eumetazoos de $A$. porosus. Debido a que el tamaño corporal de los hospedadores de una muestra suele variar, se determinó si el tamaño corporal de los bagres de mar era importante en las variaciones de los descriptores infracomunitarios (riqueza, abundancia numérica y volumétrica, dominancia, diversidad y uniformidad de parásitos) mediante correlaciones entre variables. La abundancia volumétrica de parásitos, también referida como biovolumen, ha sido considerada sólo recientemente (George-Nascimento et al. 2002) por lo que ha sido poco aplicada. Este descriptor podría ser muy útil en el análisis de las infracomunidades, ya que el tamaño de las especies parásitas suele ser muy distinto y, en algunos casos, la abundancia numérica de parásitos no se correlaciona con la abundancia volumétrica (George-Nascimento et al. 2002), lo que implica que los descriptores de abundancia, medida en número o en volumen, pueden ser distintos. Como objetivo secundario, este estudio indagó sobre las posibles vías de transmisión de los taxones parasitarios encontrados en el bagre de mar y se construyó una posible trama trófica donde $A$. porosus participa, la cual fue deducida a partir de los ciclos vitales generales de las especies parásitas registradas en este hospedador.

\section{Material y métodos}

Se recolectaron 30 individuos de Aphos porosus desde el intermareal rocoso de El Tabo ( $33^{\circ} 27^{\prime}$ 'S, $71^{\circ} 38^{\prime} \mathrm{O}$ ) en Chile central entre octubre 2006 y enero 2007. Los peces se capturaron siguiendo el procedimiento descrito por Díaz \& George-Nascimento (2002). La mayoría de los individuos se congelaron $(n=25)$, mientras que algunos fueron diseccionados en estado fresco. Se determinó el peso corporal de cada ejemplar, el cual se consideró como la variable de tamaño corporal de los hospedadores. Posteriormente se realizó el examen parasitológico según el método de George-Nascimento \& Iriarte (1989). Los parásitos recolectados se fijaron para su preservación en formalina al 10\% y luego se realizó la determinación 
taxonómica mediante literatura especializada (Moore 1910, Petrochenko 1971, Smith 1970). Para la identificación a nivel de género, los nemátodos anisákidos se aclararon en clorolactofenol para observar sus estructuras internas (Möller \& Anders 1986).

Para cada taxón parasitario se calculó la prevalencia (porcentaje de hospedadores en la muestra con uno o más individuos de una especie particular de parásito) con su respectivo intervalo de confianza al 95\% y la abundancia (promedio de individuos para cada especie parásita en el total de hospedadores examinados) (Bush et al. 1997). También se calculó la abundancia volumétrica de parásitos, que se obtiene de la multiplicación entre la abundancia numérica de una especie parásita y su volumen corporal (George-Nascimento et al. 2002). Para ello se midió el largo y ancho del cuerpo de cada taxón parasitario para estimar su volumen corporal mediante la aplicación de fórmulas matemáticas según la forma geométrica del parásito. La unidad de medida de este descriptor fue $\mathrm{mm}^{3}$ de parásitos.

A nivel infracomunitario, los descriptores parasitológicos utilizados fueron la riqueza (número de taxones parásitos por hospedador), abundancia total (número de individuos parásitos de todas las especies por individuo hospedador) (Bush et al. 1997), dominancia y abundancia volumétrica $\left(\mathrm{mm}^{3}\right.$ de parásitos considerando a todas las especies parásitas por individuo hospedador) (George-Nascimento et al. 2002). La diversidad y la uniformidad (= 'eveness') se calculó mediante el índice de Brillouin (Magurran 1988). Para determinar si el peso corporal del bagre de mar es importante para entender las variaciones de los descriptores poblacionales y comunitarios de parásitos, se calcularon los coeficientes de correlación de Spearman $\left(\mathrm{r}_{\mathrm{s}}\right)$ y su significancia estadística (Zar 1996).

Una vez determinada la composición de especies parásitas de Aphos porosus, se construyó una posible red trófica en la que este pez participaría de acuerdo a los taxones parásitos identificados. Para ello se utilizó información bibliográfica sobre los ciclos de vida generales de los parásitos y sobre los grupos de especies que interaccionan tróficamente con el bagre de mar.

\section{Resultados}

Toda la muestra estuvo compuesta por bagres de mar maduros, 26 fueron machos y 4 fueron hembras. El peso corporal promedio de estos hospedadores fue de 238,6 \pm 96,6 g correspondiente a 26,8 $\pm 3,9 \mathrm{~cm}$ de longitud total. Las hembras tuvieron menor peso corporal que los machos (96,6 $\pm 15,3$ g y 251,7 $\pm 88,3$ g respectivamente).
La muestra de hospedadores no fue analizada por sexo debido al bajo número de hembras obtenido. El 93,3\% de los ejemplares examinados albergaba al menos un taxón parasitario. De los 28 hospedadores parasitados, se recolectaron 2.104 parásitos y se identificaron nueve taxones (Fig. 1), siendo la sanguijuela Platybdella chilensis Moore, 1910 el único taxón ectoparásito encontrado (Tabla 1). La abundancia promedio de la muestra fue de 70,1 \pm 71,5 individuos parásitos por hospedador y la riqueza promedio fue de 2,4 $\pm 1,4$ especies por individuo hospedador (Tabla 2).

Las larvas de cestodos Tetraphyllidea y el nemátodo Pseudoterranova sp. fueron los parásitos más prevalentes y abundantes en número (Tabla 1). En prevalencia les siguieron Anisakis sp. y Clestobothrium crassiceps (Rudolphi, 1819), y en abundancia numérica, Corynosoma sp. y C. crassiceps. La mayor abundancia volumétrica promedio de parásitos ocurrió con el nemátodo Pseudoterranova sp. seguido por los cestodos Tetraphyllidea, pero con valores muy inferiores al obtenido por los nemátodos (Tabla 1).

La abundancia numérica y volumétrica de parásitos aumentaron significativamente con el peso corporal del hospedador (Tabla 2) y la riqueza de especies parásitas se correlacionó positivamente con esta variable, pero con un valor cercano a la probabilidad crítica de 0,05. La dominancia, diversidad de Brillouin y la uniformidad de parásitos no mostraron correlaciones significativas con el tamaño corporal del hospedador (Tabla 2).

Las abundancia numérica y volumétrica de Tetraphyllidea $\left(\mathrm{r}_{\mathrm{s}}=0,46, P=0,010 \mathrm{y} \mathrm{r}_{\mathrm{s}}=0,47, P=0,009\right.$ respectivamente) aumentaron significativamente con el tamaño del hospedador, mientras que ninguna de las abundancias de Pseudoterranova sp. se correlacionó significativamente con el peso corporal del hospedador (numérica: $\mathrm{r}_{\mathrm{s}}=0,30, P=0,108$ y volumétrica: $\mathrm{r}_{\mathrm{s}}=0,32$ y $P=0,08$ ).

Todos los endoparásitos recolectados desde las infracomunidades parasitarias del bagre de mar se encontraban en estado larval, por lo que el bagre de mar sería hospedador intermediario o paraténico, según el caso, para todas las especies de cestodos, nemátodos y acantocéfalos, los cuales alcanzan su madurez sexual en elasmobranquios o mamíferos marinos. Los cestodos fueron encontrados en estados de larva plerocercoides para los Tetraphyllidea y plerocercus para los Trypanorhyncha (Nybelinia sp. y Grillotia sp.), lo que implica que el bagre de mar es el hospedador intermediario para estos parásitos. Corynosoma sp. y Polymorphus sp. fueron encontrados en estado de cistacantos, lo que ubicaría al bagre de mar como hospedador paraténico para estos acantocéfalos. Los nemátodos anisákidos, Anisakis 
sp. y Pseudoterranova sp. estuvieron en estado de $\mathrm{L}_{3}$, siendo Aphos porosus un hospedador intermediario o paraténico (Fig. 2) según sea la ruta de transmisión.

\section{Discusión}

La fauna parasitaria encontrada en los ejemplares de Aphos porosus utilizados en este estudio corresponde al primer catastro de todos los parásitos eumetazoos (endo y ectoparásitos) en el bagre de mar. Previamente existía sólo un registro de endoparásitos para esta especie en ríos y estuarios del sur de Chile (Torres et al. 1993). Los cestodos Tetraphyllidea (identificados como $S$. pleuronectis por Torres et al. (1993)), los nemátodos anisákidos y el acantocéfalo Corynosoma sp. serían parásitos comunes del bagre de mar.

A pesar de las diferencias de tamaño corporal de las especies parásitas, esto no influyó en la correlación de la abundancia numérica y volumétrica con el tamaño corporal del hospedador, ya que ambos descriptores aumentaron significativamente con el peso corporal de los bagres de

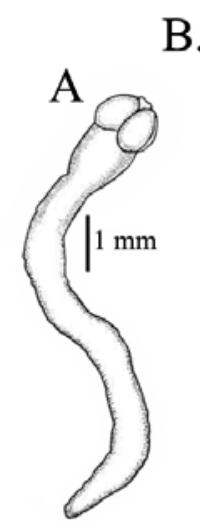

B. $1_{\oplus}$
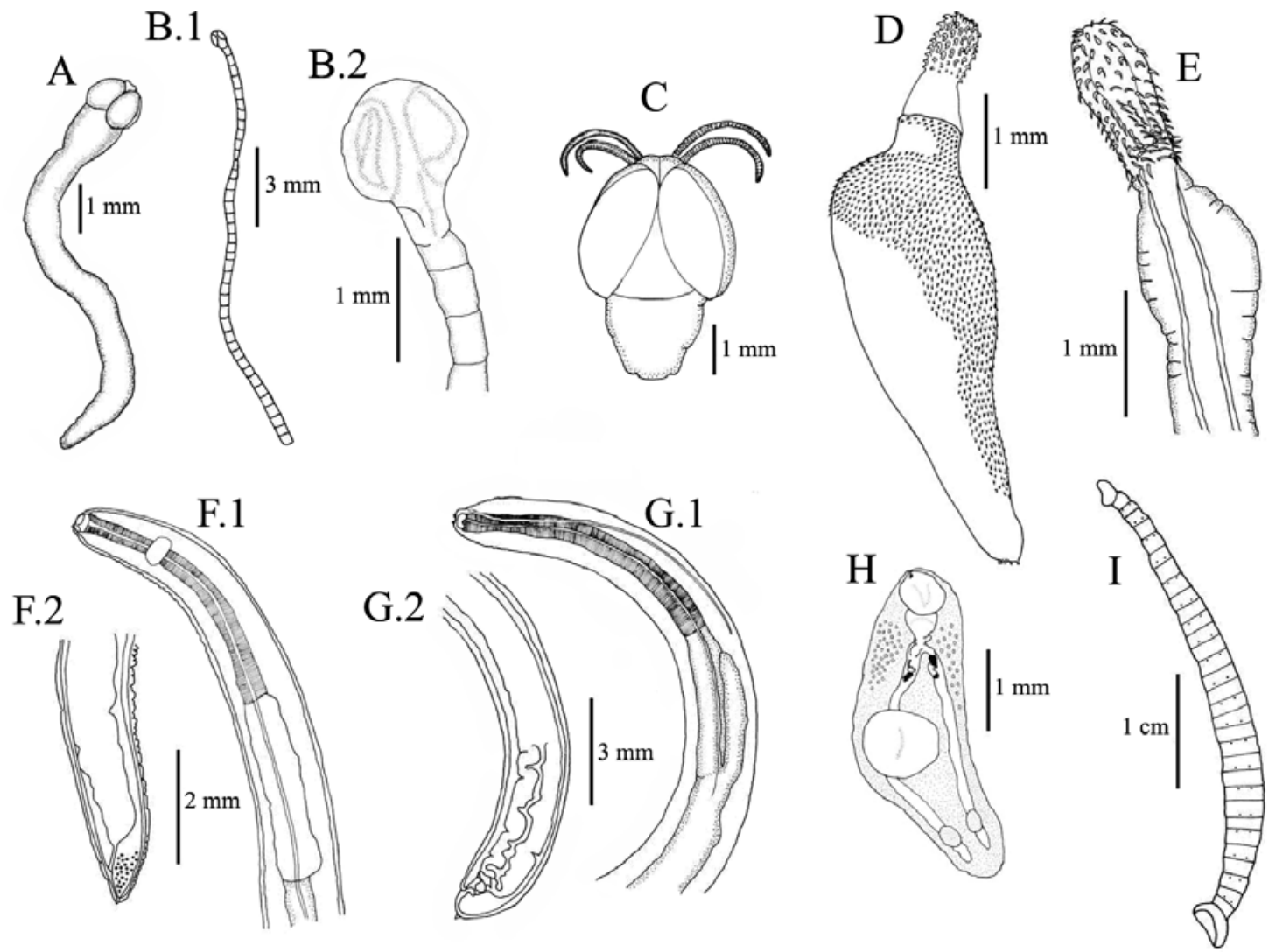

Figura 1

Taxones de parásitos encontrados en el bagre de mar Aphos porosus. (A) Tetraphyllidea; (B.1) cuerpo entero y (B.2) escólex de Clestobothrium crassiceps.; (C) Nybelinia sp.; (D) Corynosoma sp., (E) Polymorphus sp.; (F.1) región anterior y (F.2) posterior de Anisakis sp.; (G.1) región anterior y (G.2) posterior de Pseudoterranova sp.; (H) Proctoeces lintoni; (I) Platybdella chilensis

Taxa of parasites found in the toadfish Aphos porosus. (A) Tetraphyllidea; (B.1) whole body and (B.2) scolex of Clestobothrium crassiceps; (C) Nybelinia sp.; (D) Corynosoma sp. (E) Polymorphus sp.; (F.1) anterior and (F.2) posterior regions of Anisakis sp.; (G.1) anterior and (G.2) posterior regions of Pseudoterranova sp.; (H) Proctoeces lintoni; (I) Platybdella chilensis 


\section{Tabla 1}

Especies parásitas de Aphos porosus. Localización en el cuerpo del hospedador, número total de individuos parásitos por taxón, prevalencia (P\%) y su intervalo de confianza al 95\% (IC), media de abundancia numérica (AN) y volumétrica

$\left(\mathrm{AV}, \mathrm{mm}^{3}\right)$ de parásitos con sus respectivas desviaciones estándar $(\mathrm{DE}) . \mathrm{I}=$ intestino, $\mathrm{CC}=$ cavidad celomática, M = musculatura, $\mathbf{B}=$ branquias, $\mathbf{G}$ = gónadas, $\mathrm{SC}=$ superficie corporal

Parasite species of Aphos porosus. Localization of the parasites in the host body, number of parasites found in each taxon, prevalence (P, expressed in \%) and confidence intervals for prevalence at 95\% (IC), averages of numeric abundance (AN) and volumetric abundance $\left(\mathrm{AV}, \mathrm{mm}^{3}\right)$ of parasites and standard deviation $(\mathrm{DE})$ for each descriptor. $\mathrm{I}=$ intestine,

CC = celomatic cavity, $\mathrm{M}$ = muscles, $\mathrm{B}$ = gills, $\mathrm{G}$ = gonads, $\mathrm{SC}$ = body surface

\begin{tabular}{|c|c|c|c|c|c|c|}
\hline Especies parásitas & & Localización & $\mathrm{N}^{\circ}$ total & $\mathrm{P} \% \pm \mathrm{IC}$ & $\mathrm{AN}(\mathrm{DE})$ & AV (DE) \\
\hline Trematoda & Proctoeces lintoni $\uparrow$ & I & 1 & $3,3 \pm 6,4$ & $0,03(0,18)$ & $0,002(---)$ \\
\hline \multirow[t]{3}{*}{ Cestoda } & Tetraphyllidea * & I & 1.920 & $80,0 \pm 14,3$ & $64,00(67,99)$ & $6,272(0,06)$ \\
\hline & $\begin{array}{l}\text { Clestobothrium } \\
\text { crassiceps } * \dagger\end{array}$ & I & 9 & $23,3 \pm 15,3$ & $0,30(0,60)$ & $0,164(0,29)$ \\
\hline & Nybelinia sp.* & $\mathrm{CC}$ & 3 & $6,7 \pm 8,9$ & $0,10(0,40)$ & $0,011(0,02)$ \\
\hline \multirow[t]{2}{*}{ Acanthocephala } & Polymorphus sp.* & $\mathrm{CC}$ & 1 & $3,3 \pm 6,4$ & $0,03(0,18)$ & 0,007 (---) \\
\hline & Corynosoma sp. ${ }^{*}$ & $\mathrm{CC}$ & 28 & $13,3 \pm 12,2$ & $0,93(4,38)$ & $0,386(0,86)$ \\
\hline \multirow[t]{2}{*}{ Nematoda } & Anisakis sp. * & $\mathrm{M}, \mathrm{CC}$ & 10 & $23,3 \pm 15,1$ & $0,33(0,66)$ & $0,952(1,47)$ \\
\hline & Pseudoterranova sp. * & $\mathrm{M}, \mathrm{CC}, \mathrm{B}, \mathrm{G}$ & 128 & $80,0 \pm 14,3$ & $4,27(5,48)$ & $28,794(3,05)$ \\
\hline Hirudinea & Platybdella chilensis $\S$ & $\mathrm{SC}$ & 4 & $10,0 \pm 10,7$ & $0,13(0,43)$ & $1,462(2,22)$ \\
\hline
\end{tabular}

Estado de desarrollo: * larval, † inmaduro, § adulto. La especie de sanguijuela fue pobremente descrita (Moore 1910), por lo que recientemente se ha sugerido que podría corresponder al género Austrobdella (Williams et al. 2007).

mar. Los factores que podrían afectar estas correlaciones positivas son la cantidad de presas infectadas consumidas por el pez, la tasa de transmisión de parásitos, los factores físicos del ambiente que influyen en el reclutamiento de parásitos (Sapp \& Esch 1994) o la acumulación de parásitos en el hospedador, ya que nemátodos y acantocéfalos se encontraban en la cavidad celomática o musculatura del bagre de mar desde donde no podría haber pérdida de parásitos. Sin embargo, otros factores como el tamaño muestreal y rango de tamaño corporal del hospedador también podrían afectar estas correlaciones (e.g., contrastar datos de Balboa \& George-Nascimento 1998 y González \&Acuña 2000), de hecho los peces obtenidos en este estudio eran adultos y estaban en un rango estrecho de pesos corporales, pudiendo ser este factor el causante de las bajas correlaciones para los otros descriptores parasitológicos (riqueza, dominancia, diversidad y uniformidad). En todo caso, la influencia del tamaño corporal del hospedador en los descriptores infrapoblacionales e infracomunitarios de parásitos suele ser variable en el tiempo y entre especies hospedadoras, debido a los múltiples factores implicados en los procesos de transmisión de parásitos.
Tabla 2

Promedio (X) y desviación estándar (DE) de seis descriptores infracomunitarios de parásitos. Correlación de Spearman entre los descriptores y el peso corporal del bagre de mar Aphos porosus; $n=30, r_{s}$ : coeficiente de correlación de Spearman, $P$ : probabilidad estadística, nivel de significancia 0,05

Average (X) an d standard deviation (DE) of six infracommunity descriptors of parasites. Spearman correlation between these descriptors and body weight of the toadfish Aphos porosus; n= 30, $\mathrm{r}_{\mathrm{s}}$ : Spearman's rank correlation coefficient, $P$ : statistic probability, significant level $=0.05$

\begin{tabular}{lccc}
\hline Descriptor comunitario & $\mathrm{X}(\mathrm{DE})$ & $\mathrm{r}_{\mathrm{S}}$ & $P$ \\
\hline Abundancia numérica & $70,13(71,45)$ & 0,46 & $0,011^{*}$ \\
Abundancia volumétrica & $40,72(45,19)$ & 0,38 & $0,039^{*}$ \\
Riqueza de especies & $2,43(1,43)$ & 0,35 & 0,060 \\
Dominancia & $0,80(0,26)$ & 0,21 & 0,256 \\
Diversidad de Brillouin & $0,21(0,23)$ & 0,05 & 0,809 \\
Uniformidad & $0,21(0,24)$ & 0,08 & 0,676
\end{tabular}

* correlaciones estadísticamente significativas 
Vol. 43, N², 2008

Hospedadores definitivos

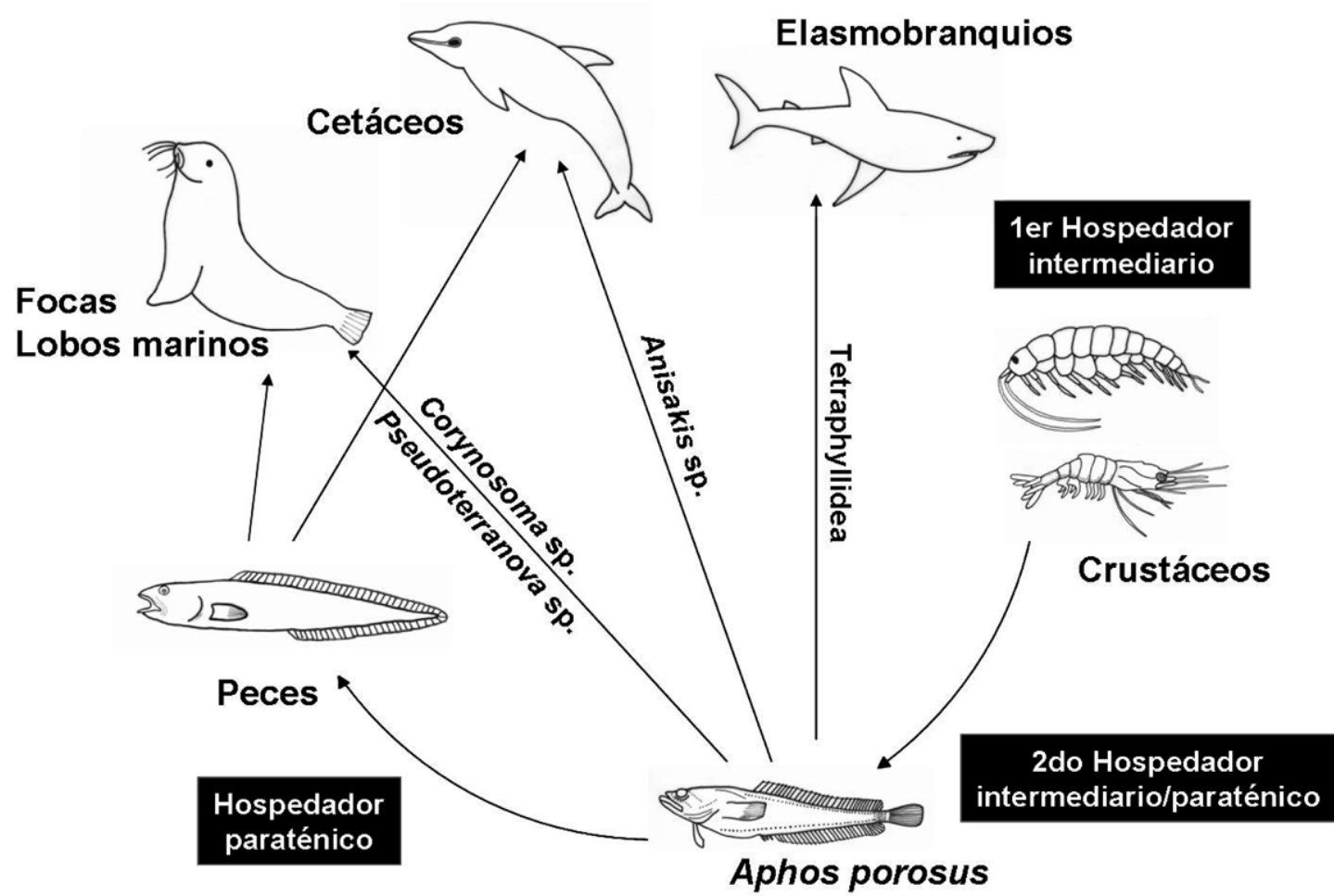

Figura 2

Función del bagre de mar Aphos porosus en las vías de transmisión de parásitos en Chile central

Role of the toadfish Aphos porosus in the modes of transmission of parasites in central Chile

El bagre de mar presentó una baja riqueza de ectoparásitos compuesta sólo por la sanguijuela $P$. chilensis. La explicación podría estar relacionada con la gran cantidad de mucus que produce el bagre de mar, el cual constituye la barrera inmune del hospedador en cuya composición hay productos que pueden hacer inhóspita la superficie corporal para algunas especies parásitas (Whittington et al. 2000). Por lo tanto, pocos parásitos podrían estar adaptados para vivir en la superficie del bagre de mar.

La composición de las comunidades de parásitos de Aphos porosus no es similar a la de los peces intermareales, como Gobiesox marmoratus Jenyns, 1842 (Pardo-Gandarillas et al. 2004), Scartichthys viridis (Valenciennes, 1836) (Díaz \& George-Nascimento 2002), Bovichtus chilensis Regan, 1913 (Muñoz et al. 2002). Todos los peces intermareales básicamente se caracterizan porque sus comunidades de parásitos están conformadas principalmente por copépodos y tremátodos, parásitos ausentes en el bagre de mar, salvo Proctoeces lintoni Siddiqui \& Cable, 1960 (Tabla 1) que es un parásito común del pejesapo Sicyases sanguineus Müller \& Troschel, 1843 (Oliva 1984) el cual habita en el intermareal rocoso. Sin embargo, este digeneo fue encontrado en el bagre de mar en muy baja frecuencia y en estado inmaduro. En contraste, la composición parasitaria del bagre de mar es más similar a la de peces demersales, ya que comparte con ellos varios grupos taxonómicos, como nemátodos (Anisakis sp. y Pseudoterranova sp.), cestodos (Trypanorhyncha y Tetraphyllidea) y acantocéfalos (Corynosoma sp.). De hecho, el bagre de mar comparte al menos dos taxones parasitarios con la cabrilla $S$. capensis (González \& Acuña 2000), la merluza de cola Macruronus magellanicus Lönnberg, 1907 (Torres et al. 1993), la 
merluza austral Merluccius australis (Hutton, 1872) (George-Nascimento \& Arancibia 1994), la merluza común M. gayi (Guichenot, 1848) (Oliva \& Ballón 2002) y el blanquillo P. jugularis (Sepúlveda et al. 2004).

Los bagres de mar analizados en este estudio no presentaron contenido estomacal, lo cual es coincidente con los resultados obtenidos por Varas \& Ojeda (1990). Este resultado parece indicar que este pez no se alimenta o se alimenta poco durante su estadía en el área intermareal, y resulta importante ya que la mayoría de los endoparásitos son transmitidos por la dieta, por lo que $A$. porosus tendría escasas oportunidades de adquirir nuevas especies parásitas características del ambiente intermareal. La semejanza de parásitos con peces demersales sugiere que conservaría sus especies parásitas y estas no se perderían cuando habita temporalmente el intermareal probablemente por la ubicación de los parásitos en el cuerpo del hospedador. Por lo tanto, el bagre de mar conserva la mayoría de sus parásitos y prácticamente no adquiere nuevas especies durante su estadía en el intermareal.

Todos los endoparásitos encontrados en el bagre de mar tendrían ciclos de vida indirectos compuestos por al menos tres hospedadores: intermediarios (crustáceos y el bagre de mar), paraténico (bagre de mar) y definitivos (elasmobranquios y mamíferos marinos) (Fig. 2). Esta evidencia sugiere que $A$. porosus depreda sobre crustáceos y a su vez es depredado por otros peces demersales, elasmobranquios o mamíferos marinos, por lo que sería un consumidor de nivel trófico intermedio (consumidor secundario) dentro de la red del ecosistema demersal.

El bagre de mar sería hospedador paraténico para las larvas de acantocéfalos, Corynosoma sp. y Polymorphus sp., los cuales alcanzan su madurez sexual en mamíferos marinos como los otáridos (lobos marinos) (GeorgeNascimento \& Carvajal 1981) y cetáceos (Torres et al. 1992) respectivamente. Mientras que sería un hospedador intermediario para Anisakis sp., Pseudoterranova sp. y Tetraphyllidea. La presencia de estos parásitos indicaría que el bagre de mar puede ser depredado alternativamente por cetáceos, otáridos (hospedadores definitivos para Anisakis y Pseudoterranova) (George-Nascimento \& Carvajal 1980, 1981, George-Nascimento \& Vergara 1981, Torres et al. 1993, Mercado et al. 1997) o elasmobranquios (hospedadores definitivos para Tetraphyllidea) (Carvajal 1974).

La presencia de Anisakis sp., Pseudoterranova sp. y Tetraphyllidea además indica que el bagre de mar depreda sobre crustáceos, posiblemente al camarón Heterocarpus reedii Bahamonde, 1955 y la jaiba Cancer plebejus
Poeppig, 1836, los cuales han sido determinados como hospedadores intermediarios para los nemátodos (George-Nascimento \& Carvajal 1980, GeorgeNascimento et al. 1994). Mientras que larvas Tetraphyllidea suelen tener como hospedadores intermediaros a diversos crustáceos, aunque en Chile no hay antecedentes al respecto. Cabe mencionar que algunas especies de Tetraphyllidea podrían tener sus estados de plerocercoides o merocercoides en moluscos bivalvos (Carvajal \& Mellado 2007), aunque en esos casos, las vías de transmisión que incluyen a moluscos no tienen a peces como segundo hospedador intermediario. Hasta el momento, A. porosus es el único hospedador con una alta cantidad de larvas tetrafilideas y en altas prevalencias según lo observado en otros estudios (George-Nascimento et al. 2002, Oliva \& Ballón 2002, Sepúlveda et al. 2004), siendo este taxón dominante entre las otras especies parásitas encontradas en el bagre de mar, lo que indicaría que este pez es un hospedador recurrente para la transmisión de estos cestodos.

En Chile se han reportado cerca de 18 especies de peces, entre ellas el bagre de mar como hospedadoras del tercer estado larval de Pseudoterranova sp. (Mercado et al. 1997). Esta larva, al igual que la $\mathrm{L}_{3}$ de Anisakis sp., es encapsulada en la musculatura de los hospedadores de transporte sin desarrollarse al siguiente estado $\left(\mathrm{L}_{4}\right.$, si la hubiere (Pereira 1992), o adulto), mientras que en los hospedadores definitivos se desarrollan en el tracto digestivo (Pereira 1992). Los mamíferos marinos, que son los hospedadores definitivos de los anisákidos, aparentemente no están en contacto con los pequeños crustáceos (e.g., decápodos, anfípodos, larvas de crustáceos) los cuales han sido indicados en la literatura como hospedadores intermediarios de estos parásitos. Los anisákidos pueden tomar variadas rutas de transmisión, implicando a uno o dos hospedadores invertebrados, uno o dos peces como hospedadores intermediarios (a veces uno es obligatorio) y uno o dos hospedadores paraténicos (Klimpel et al. 2004). Por lo tanto, el bagre de mar podría actuar como hospedador intermediario o paraténico para los nemátodos, dependiendo de la ruta de transmisión que tenga el parásito. No hay registros específicos de que lobos marinos o cetáceos tengan en sus dietas a los bagres de mar, pero sí hay registros de otros peces teleósteos (e.g., congrios) que depredan sobre el bagre de mar (Delfin 1903) y que son consumidos por lobos marinos (GeorgeNascimento et al. 1985), por lo tanto, es posible sugerir que el siguiente hospedador pueda ser un pez, que actuaría claramente como un hospedador paraténico para los anisákidos (Fig. 2). 
En este estudio no sólo se ha cuantificado la comunidad de parásitos de $A$. porosus, sino que además se ha inferido sobre el rol del bagre de mar en la transmisión de sus parásitos entre otros hospedadores, lo que ha permitido indagar sobre las vías de transmisión de los parásitos hacia el bagre de mar, desde sus potenciales presas, y hacia los hospedadores definitivos, depredadores del bagre de mar. De este modo, este estudio destaca los alcances del parasitismo al deducir las redes alimentarias y las interacciones entre las poblaciones de un pez, las de sus depredadores y las de sus presas (Marcogliese 2003), todos ellos componentes esenciales en la transmisión de parásitos.

\section{Agradecimientos}

Agradecemos a Leonardo Zamora, Natalí Delorme y Karen Flores por su ayuda en terreno y al financiamiento del Proyecto FONDECYT 11060006. También agradecemos las críticas y sugerencias de tres evaluadores anónimos, los que permitiron mejorar el presente manuscrito.

\section{Literatura citada}

Angel A \& F Ojeda. 2001. Structure and trophic organization of subtidal fish assemblages on the northern Chilean coast: the effect of habitat complexity. Marine Ecology Progress Series 217: 81-91.

Balboa L \& M George-Nascimento. 1998. Variaciones ontogenéticas y entre años en las infracomunidades de parásitos metazoos de dos especies de peces marinos de Chile. Revista Chilena de Historia Natural 71: 27-37.

Bush A, K Lafferty, J Lotz \& A Shostak. 1997. Parasitology meets ecology on its own terms: Margolis et al. revisited. Journal of Parasitology 83: 575-583.

Carvajal J. 1974. Records of cestodes from Chilean sharks. Journal of Parasitology 60: 29-34.

Carvajal J \& A Mellado. 2007. Utilización de la morfología de las larvas merocercoides presentes en moluscos, en la dilucidación de la taxonomía de las especies Rhodobothrium (Cestoda: Tetraphyllidea). Gayana 71: 114-119.

Chirichigno N. 1974. Clave para identificar a los peces marinos del Perú. Instituto del Mar del Perú 44: 1-387.

Delfin F. 1903. Los congrios de Chile. Revista Chilena de Historia Natural 7: 154-192.

Díaz F \& M George-Nascimento. 2002. Estabilidad temporal de las infracomunidades de parásitos en la borrachilla Scartichthys viridis (Valenciennes, 1836) (Pisces: Blenniidae) en la costa central de Chile. Revista Chilena de Historia Natural 75: 641-649.

George-Nascimento M. 1987. Ecological helminthology of wildlife animal hosts from South America: a literature review and search for patterns in marine food webs. Revista Chilena de Historia Natural 60: 187-202.
George-Nascimento M \& H Arancibia. 1994. La fauna parasitaria y la morfometría de la merluza austral Merluccius australis (Hutton) como indicadoras de unidades de stock. Biología Pesquera 23: 31-47.

George-Nascimento M, R Bustamante \& C Oyarzún. 1985. Feeding ecology of the South American sea lion Otaria flavescens: food contents and food selectivity. Marine Ecology Progress Series 21: 135-143.

George-Nascimento M, R Carmona \& R Riffo. 1994. Occurrence of larval nematodes Proleptus sp. (Spirurida: Physalopteridae) and Anisakis sp. (Ascaridida: Anisakidae) in the crab Cancer plebejus Poeppig, in Chile. Scientia Marina 58: 355-358.

George-Nascimento M \& J Carvajal. 1980. Nuevos registros de nematodos anisákidos en la fauna marina chilena. Boletín Chileno de Parasitología 35: 15-18.

George-Nascimento M \& J Carvajal. 1981. Helmintos parásitos del lobo marino común Otaria flavescens en el Golfo de Arauco, Chile. Boletín Chileno de Parasitología 36: 72-73.

George-Nascimento M, F Garcías \& G Muñoz. 2002. Parasite body volume and infracommunity patterns in the southern pomfret Brama australis (Pisces: Bramidae). Revista Chilena de Historia Natural 75: 835-839.

George-Nascimento $\mathbf{M} \quad \& \quad J$ Iriarte. 1989. Las infracomunidades de parásitos metazoos del chancharro Helicolenus lengerichi Norman, 1937 (Pisces, Scorpaenidae): un ensamble no interactivo de peces. Revista Chilena de Historia Natural 62: 217-227.

George-Nascimento M \& L Vergara. 1981. Balaenoptera borealis Lesson, 1828, primer registro de huésped definitivo para Anisakis simplex (Rudolphi, 1809, det. Krabbe, 1887) en Chile. Parasitología al Día 5: 91-92.

González M \& E Acuña. 2000. Influence of host size and sex on the endohelminth infracommunities of the red rockfish Sebastes capensis off Northern Chile. Journal of Parasitology 86: 854-857.

González M, C Barrientos \& C Moreno. 2006. Biogeographical patterns in endoparasite communities of marine fish (Sebastes capensis Gmelin) with extended range in the Southern Hemisphere. Journal of Biogeography 33: 1086-1095.

González M \& C Moreno. 2005. The distribution of the ectoparasite fauna of Sebastes capensis from the southern hemisphere does not correspond with zoogeographical provinces of free-living marine animals. Journal of Biogeography 32: 1539-1547.

Klimpel S, H Palm, S Ruckert \& U Piatkowski. 2004. The life cycle of Anisakis simplex in the Norwegian Deep (northern North Sea). Parasitology Research 94: 1-9.

Magurran AE. 1988. Ecological diversity and its measurements, 179 pp. Croom Helm, London.

Marcogliese DJ. 2003. Food webs and biodiversity: are parasites the missing link? Journal of Parasitology 89: S106S113. 
Medina M, M Araya \& A Vega. 2004. Alimentación y relaciones tróficas de peces costeros de la zona del norte de Chile. Investigaciones Marinas 32: 33-47.

Mercado R, P Torres \& J Maira. 1997. Human case of gastric infection by a fourth larval stage of Pseudoterranova decipiens (Nematoda, Anisakidae). Journal of Public Health 31: 178-181.

Möller H \& K Anders. 1986. Diseases and parasites of marine fishes, 365 pp. Verlag Möller, Kiel.

Moore J. 1910. Platybdella chilensis, sp. nov. Revista Chilena de Historia Natural 14: 29-30.

Moreno C \& J Castilla. 1975. Guía para el reconocimiento y observación de peces de Chile, 120 pp. Editora Nacional Gabriela Mistral, Santiago.

Muñoz G, V Valdebenito \& M George-Nascimento. 2002. La dieta y la fauna de parásitos metazoos del torito Bovichthys chilensis Regan 1914 (Pisces: Bovichthydae) en la costa de Chile centro-sur: variaciones geográficas y ontogenéticas. Revista Chilena de Historia Natural 75: 661671.

Oliva M. 1984. Nuevos registros de trematodos digeneos en peces marinos de Antofagasta. Ciencia y Tecnología del Mar, CONA 8: 9-15.

Oliva M \& I Ballón. 2002. Metazoan parasites of the Chilean hake Merluccius gayi gayi as a tool for stock discrimination. Fisheries Research 56: 313-320.

Oliva M, M González \& E Acuña. 2004. Metazoan parasite fauna as a biological tag for the habitat of the flounder Hippoglossina macrops from northern Chile, in a depth gradient. Journal of Parasitology 90: 1374-1377.

Oliva M \& M González. 2005. The decay of similarity over geographical distance in parasite communities of marine fishes. Journal of Biogeography 32: 1327-1332.

Pardo-Gandarillas M, F Garcías \& M George-Nascimento. 2004. La dieta y la fauna de endoparásitos del pejesapo Gobiesox marmoratus Jenyns, 1842 (Pisces: Gobiesocidae) en el litoral de Chile están conectadas pero no correlacionadas. Revista Chilena de Historia Natural 77: 627-637.

Pequeño G. 1989. Peces de Chile: Lista sistemática revisada y comentada. Revista de Biología Marina 24: 1-132.
Pereira J. 1992. Algunos aspectos de la epidemiología y prevención de la anisakiosis, 52 pp. Junta de Castilla y León. Consejería de Sanidad y Bienestar Social. Dirección General de Salud Pública, Valladolid.

Petrochenko VI. 1971. Acanthocephala of domestic and wild animals, Volumen II, 478 pp. Skryabin KI (ed), The Israel Program for Scientific Translations, Jerusalem.

Sapp K \& G Esch. 1994. The effects of spatial and temporal heterogeneity as structuring forces for parasite communities in Helisoma anceps and Physa gyrina. American Midland Naturalist 132: 91-103.

Sepúlveda F, S Marin \& J Carvajal. 2004. Metazoan parasites in wild fish and farmed salmon from aquaculture sites in southern Chile. Aquaculture 235: 89-100.

Sielfeld W \& M Vargas. 1996. Composición y estructura de la ictiofauna demersal en la zona norte de Chile. Investigaciones Marinas 24: 3-17.

Smith G. 1970. How to know the tapeworms, 266 pp. WM. C. Brown Company Publishers, Iowa.

Torres P, J Oporto, L Brieva \& L Escare. 1992. Gastrointestinal helminths of the cetaceans Phocoena spinipinnis Burmeister 1865 and Cephalorhynchus eutropia Gray 1846 from the Southern Coast of Chile. Journal of Wildlife Diseases 28: 313-315.

Torres P, A Contreras, J Revenga \& N Fritz. 1993. Helminth parasites in fishes from Valdivia and Tornagaleones river estuaries in the south of Chile. Memorias do Instituto Oswaldo Cruz 88: 491-192.

Varas E \& F Ojeda. 1990. Intertidal fish assemblages of the central Chilean coast: diversity, abundance and trophic patterns. Revista de Biología Marina 25: 59-70.

Whittington I, T Cribb, T Hamwood \& J Halliday. 2000. Host-specificity of monogenean (platyhelminth) parasites: a role for anterior adhesive areas? International Journal for Parasitology 30: 305-320.

Williams J, P Urrutia \& E Burreson. 2007. Two new species of Austrobdella (Hirudinida: Piscicolidae) from Chile. Journal of Parasitology 93: 184-189.

Zar J. 1996. Biostatistical analysis, 718 pp. Prentice Hall, Englewood Cliffs. 